\title{
Cellular fatty acid-deficient Pandoraea isolated from a patient with cystic fibrosis
}

Identification of non-fermentative Gram-negative bacilli can be challenging for most clinical laboratories, partly because some taxa are not included in the databases of most commercial systems, and partly because of the non-reactive nature and slow-growing properties of some species. We present a case in which both situations applied. A 45-year-old male patient attending a cystic fibrosis (CF) clinic in Perth, Western Australia, had been infected or colonized by a member of the Pandoraea genus since 2005. Since first being isolated, the strain has been recovered in 14 out of 18 infectious exacerbations of his CF. In our laboratory non-fermentative bacteria isolated from CF patients are subject to extensive polyphasic testing using conventional biochemical, chemotaxonomic and molecular methods (Moore et al., 2002; Segonds et al., 2003). The latter includes $16 S$ rRNA sequencing and recA PCR to identify members of the Burkholderia cepacia complex. Initial biochemical testing by commercial methods failed to provide a satisfactory identification. The Vitek 1 (bioMeriéux) identified most strains as non-fermentative Gram-negative bacillus (asaccharolytic), at a $58 \%$ confidence level, with Myroides species at a $27 \%$ confidence level as the second choice. One strain was identified as Alcaligenes faecalis at a $66 \%$ confidence level, with non-fermentative Gram-negative bacillus (asaccharolytic) at a $12 \%$ confidence level as the second choice. Identification using API 20NE strips (bioMeriéux) resulted in low discrimination after $48 \mathrm{~h}$ incubation. Confidence levels did not exceed $63 \%$ for the choices suggested by this system and taxa named included A. faecalis, Ralstonia paucula, Pseudomonas putida, Ralstonia pickettii and Achromobacter xylosoxidans.

The strains were tested also by cell-wall fatty-acid methyl ester analysis using a MIDI system (MIDI) using all libraries (RCLIN, RTSBA, RBTR and TSBA) and methods available. Results obtained with this system failed to match the strains to any taxon in the above libraries.

Conventional testing consisting of 31

biochemical and morphological

characteristics suggested that the patient's strains closely resembled members of the genus Pandoraea, as described by Coenye et al. (2000). Enzymic activity was detected by the API ZYM (bioMérieux) and matched that described by Coenye et al. (2000) for Pandoraea apista. These results were supported by $16 \mathrm{~S}$ rRNA sequencing which identified the strains as Pandoraea species, with Pandoraea pulmonicola and Pandoraea pnomenusa as the first and second choices. In addition, recA PCR results were consistently negative, eliminating the possibility that the strains may belong to the B. cepacia complex. However, our cellular fatty acid results differed greatly from those obtained by Coenye et al. (2000), Daneshvar et al. (2001) and Krejči \& Kroppenstedt (2006). The most significant feature of our strains was the total absence of lauric $(12: 0)$ and $\alpha$-hydroxylauric acids (12:0 2OH); the presence of these acids is considered a useful differential characteristic in the Pandoraea cellular fatty acid profile. Other significant differences included smaller percentages of 17:0 cyclo and 19:0 cyclo $\omega 8 \mathrm{c}$ fatty acids, and larger percentages of summed feature 2 and summed feature 3 fatty acids (Coenye et al., 2000). Summed features are a mixture of acids that cannot be separated by this system.

Before the molecular and conventional tests results were available, we did not suspect the strain to be a member of the Pandoraea as this genus had never been isolated previously from any of our CF patients. The lack of clues from the MIDI results caused confusion as the organism resembled, in morphology and antibiotic susceptibility patterns, members of the $B$. cepacia complex. There is no clear explanation as to why the strains failed to produce the two marker acids. It is possible that bacteria may in future be characterized not only in terms of their biochemical differences as biotypes or biovars, but also in terms of the presence or absence of certain cellular fatty acids. In any case, this was a timely reminder that reliance on any single laboratory method can cause seriously misleading results for non-fermentative

Gram-negative bacilli.

\section{Aravena-Román}

Division of Microbiology and Infectious Diseases, PathWest Laboratory Medicine WA, Queen Elizabeth II Medical Centre, Nedlands, WA 6009, Australia

\section{Correspondence: M. Aravena-Román (max.aravena@health.wa.gov.au)}

Coenye, T., Falsen, E., Hoste, B., Ohlen, M., Goris, J., Govan, J. R., Gillis, M. \& Vandamme, P. (2000). Description of Pandoraea gen. nov. with Pandoraea apista sp. nov., Pandoraea pulmonica sp. nov., Pandoraea sputurum sp. nov., and Pandoraea norimbergensis comb. nov. Int J Syst Evol Microbiol 50, 887-899.

Daneshvar, M. I., Hollis, D., Steigerwalt, A. G., Whitney, A. M., Spangler, L., Douglas, M. P., Jordan, J. G., MacGregor, J. P., Hill, B. C. \& other authors (2001). Assignment of CDC weak oxidizer group 2 (WO-2) to the genus Pandoraea and characterisation of three new Pandoraea genomospecies. J Clin Microbiol 39, 1819-1826.

Krejči, E. \& Kroppenstedt, R. (2006).

Differentiation of species combined into Burkholderia cepacia complex and related taxa on the basis of their fatty acid patterns. J Clin Microbiol 44, 1159-1164.

Moore, J. E., Reid, A., Millar, B. C., Jiru, X., Mccaughan, J., Goldsmith, C. E., Collins, J., Murphy, P. G. \& Elborn, J. S. (2002). Pandoraea apista isolated from a patient with cystic fibrosis: problems associated with laboratory identification. Br J Biomed Sci 59, 164-166.

Segonds, C., Paute, S. \& Chabanon, G. (2003). Use of amplified DNA restriction analysis for identification of Ralstonia and Pandoraea species: interest in determination of the respiratory bacterial flora in patients with cystic fibrosis. J Clin Microbiol 41, 3415-3418. 